\title{
The Child Maltreatment Surveillance Indicator Framework
}

\author{
Aimée Campeau, MA; Shazmeera Qadri, MPH; Farah Barakat, BA; Gabriela Williams, MSc; \\ Wendy Hovdestad, PhD; Maaz Shahid, MScPH; Tanya Lary, MA
}

Tweet this article

\begin{abstract}
The federal health portfolio has conducted surveillance on child maltreatment as a public health issue since the 1990s. The Public Health Agency of Canada (PHAC) is now releasing the Child Maltreatment Indicator Framework, to take its place alongside other PHAC frameworks, such as the Suicide Surveillance Indicator Framework. Based on a scoping review of existing reviews and meta-analyses, this Framework, along with the online interactive data tool, presents child maltreatment outcome indicators and risk and protective factors at the individual, family, community and societal levels, disaggregated by sex, age and other sociodemographic variables. This Framework will function as a valuable resource pertaining to an issue that affects at least one in three Canadian adults.
\end{abstract}

Keywords: child maltreatment, physical abuse, exposure to intimate partner violence, neglect, emotional maltreatment, sexual abuse

\section{Introduction}

Child maltreatment is a public health issue, causing both immediate and longterm physical and mental health effects. ${ }^{1}$ Surveillance data are key to policy and program efforts to prevent and address child maltreatment. The Public Health Agency of Canada (PHAC) is responsible for national surveillance of child maltreatment, including physical abuse, exposure to intimate partner violence, neglect, emotional maltreatment and sexual abuse. ${ }^{2}$ PHAC currently collects data about child maltreatment from administrative data from child welfare agencies, proxy informant surveys and self-report surveys.

The Child Maltreatment Surveillance Indicator Framework (CMSIF) gathers available data on child maltreatment outcomes as well as risk and protective factors at the individual, family, community and societal levels to support research and policy development. The CMSIF, which is being released in this issue of Health Promotion and Chronic Disease Prevention in Canada, serves as a complement to other indicator frameworks released by PHAC. These include the Suicide Surveillance Indicators (SSI), the Canadian Chronic Disease Indicators (CCDI), the Positive Mental Health Surveillance Indicator Framework (PMHSIF) and the Physical Activity, Sedentary Behaviour and Sleep (PASS) Indicator Framework.

\section{Methods}

The CMSIF was developed using a scoping review method. ${ }^{3}$ Article searches prioritized systematic reviews, meta-analyses and literature reviews that examined risk and protective factors related to any type of child maltreatment. References from retrieved article reviews were reviewed for additional citations. Relevant articles published in English between 2004 and 2016 and reports from public health organizations (e.g. the World Health Organization) were consulted. An initial list of approximately 45 to 50 indicators was reduced through consultations with child maltreatment surveillance experts as well as with the Family Violence Initiative member departments to verify that the final list of
Highlights

- The Child Maltreatment Surveillance Indicator Framework complements other indicator frameworks released by the Public Health Agency of Canada and presents available data on child maltreatment outcomes and risk and protective factors at the individual, family, community and societal level.

- One-third (34.1\%) of the Canadian population aged 15 years and older have experienced at least one type of childhood maltreatment.

- Physical abuse was experienced most often $(27.4 \%)$, followed by exposure to intimate partner violence $(10.6 \%)$ and sexual abuse $(8.1 \%)$.

- Factors such as parental mental illness, substance use and past experience of family violence can put children at higher risk of child maltreatment.

indicators was relevant and actionable within a public health approach.

\section{Results}

Data from the 2014 General Social Survey (GSS) indicate that more than one-third (34.1\%) of the population aged 15 years and older have experienced at least one of the following types of childhood maltreatment: physical or sexual abuse by an adult and/or exposure to violence by parents or guardians (Table 1). Of the three types of childhood maltreatment, physical abuse was experienced most often $(27.4 \%)$, followed by exposure to intimate partner violence $(10.6 \%)$ and sexual abuse $(8.1 \%)$. 
Because of the familial context in which most child maltreatment occurs, risk and protective factors focused on parental and/or family characteristics rather than child characteristics. Factors such as parental mental illness, substance abuse and past experience of family violence can put children at higher risk of child maltreatment.

\section{Conclusion}

Childhood experiences are a key social determinant of health. ${ }^{4}$ The CMSIF provides available evidence about contexts and risk and protective factors to help target strategies to prevent and address child maltreatment.

The CMSIF is an evergreen document; it will be reviewed periodically and updated as new data become available. An online interactive tool on the Government of Canada's Public Health InfoBase will provide breakdowns for the indicators by sex, age, province/territory, immigrant status.

The data gaps identified in the framework, particularly those at the community and societal level, are being addressed through active data development using surveys and administrative data.

\section{Conflicts of interest}

The authors have no conflicts of interest to declare.

\section{Authors' contributions and statement}

AC, SQ, GW, MS and WH were involved in the design and/or conceptualization of the work, as well as the acquisition, analysis or interpretation of the data. FB, WH and TL were involved in the interpretation of the data, as well as drafting and revising the paper.

The content and views expressed in this article are those of the authors and do not necessarily reflect those of the Government of Canada.

\section{References}

1. World Health Organization. Child maltreatment: key facts [Internet]. Geneva (CH): World Health Organization; 2016 [cited 2019 Sep 10]. Available from: https://www.who.int/news-room /fact-sheets/detail/child-maltreatment
2. Public Health Agency of Canada. Child maltreatment in Canada [Internet]. Ottawa (ON): Public Health Agency of Canada; 2012 [modified 2012 Jul 26; cited 2019 Sep 10]. Available from: http://www.phac-aspc.gc.ca/sfv-avf /sources/nfnts/nfnts-2006-maltr /index-eng.php

3. Ganann R, Ciliska D, Thomas H. Expediting systematic reviews: methods and implications of rapid reviews. Implement Sci. 2010;5:56-66. doi: 10.1186/1748-5908-5-56.

4. Public Health Agency of Canada. Social determinants of health and health inequalities [Internet]. Ottawa (ON): Government of Canada; 2018 [modified 2019 Jun 28; cited 2019 Sep 10]. Available from: https://www .canada.ca/en/public-health/services /health-promotion/population-health /what-determines-health.html

5. Statistics Canada. Family violence in Canada: a statistical profile, 2015. Ottawa (ON): Statistics Canada; [modified 2017 May 4; cited 2019 Sep 10]. [Statistics Canada, Catalogue No.: 85002-X].

6. Walsh CA, MacMillan HL, Trocmé N, Jamieson E, Boyle MH. Measurement of victimization in adolescents: development and validation of the Childhood Experiences of Violence Questionnaire. Child Abuse Negl. 2008;32(11):1037-57. 
TABLE 1

\section{CHILD MALTREATMENT SURVEILLANCE INDICATOR FRAMEWORK}

\section{QUICK STATS, CANADA, 2019 EDITION}

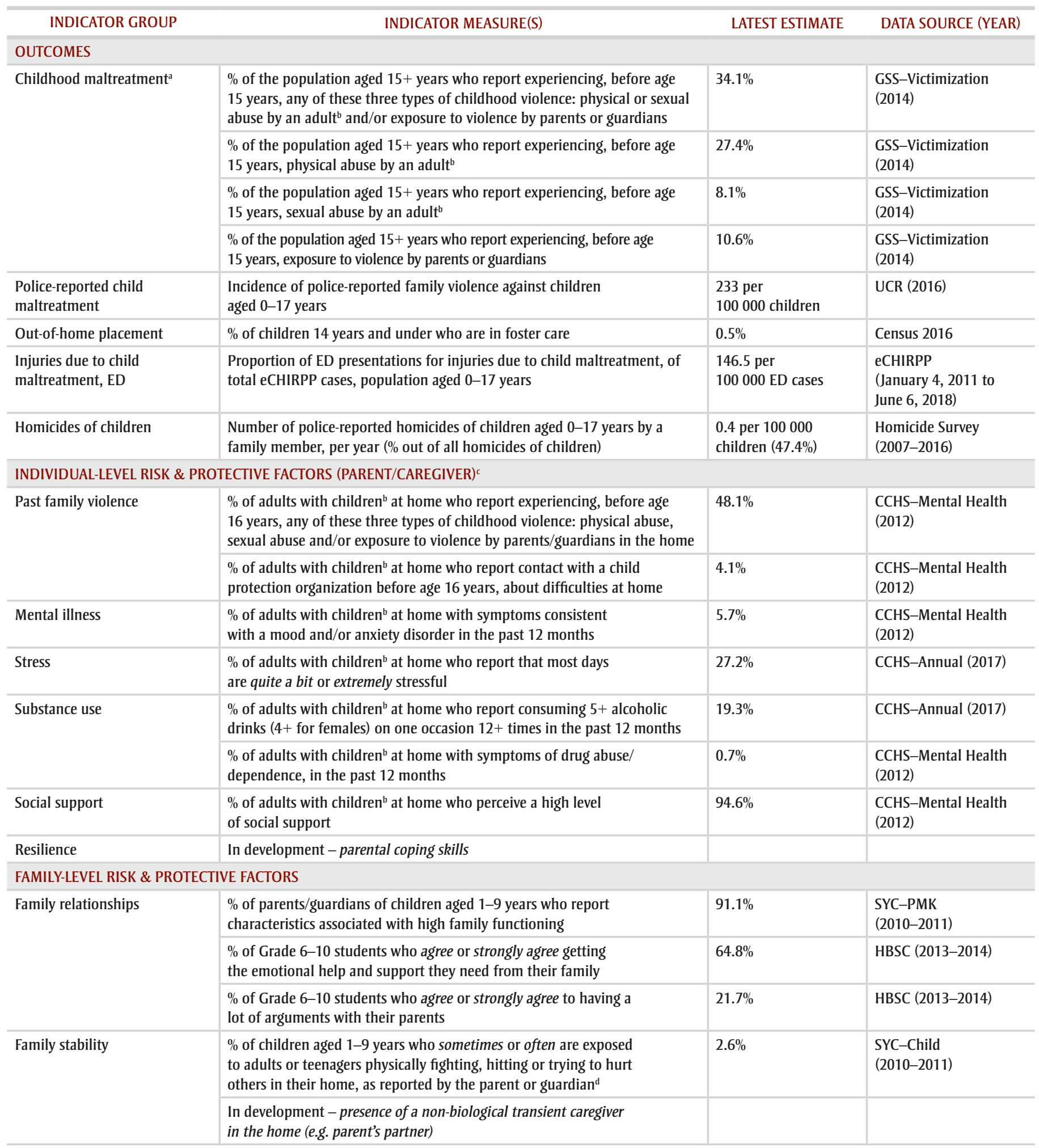

Continued on the following page 


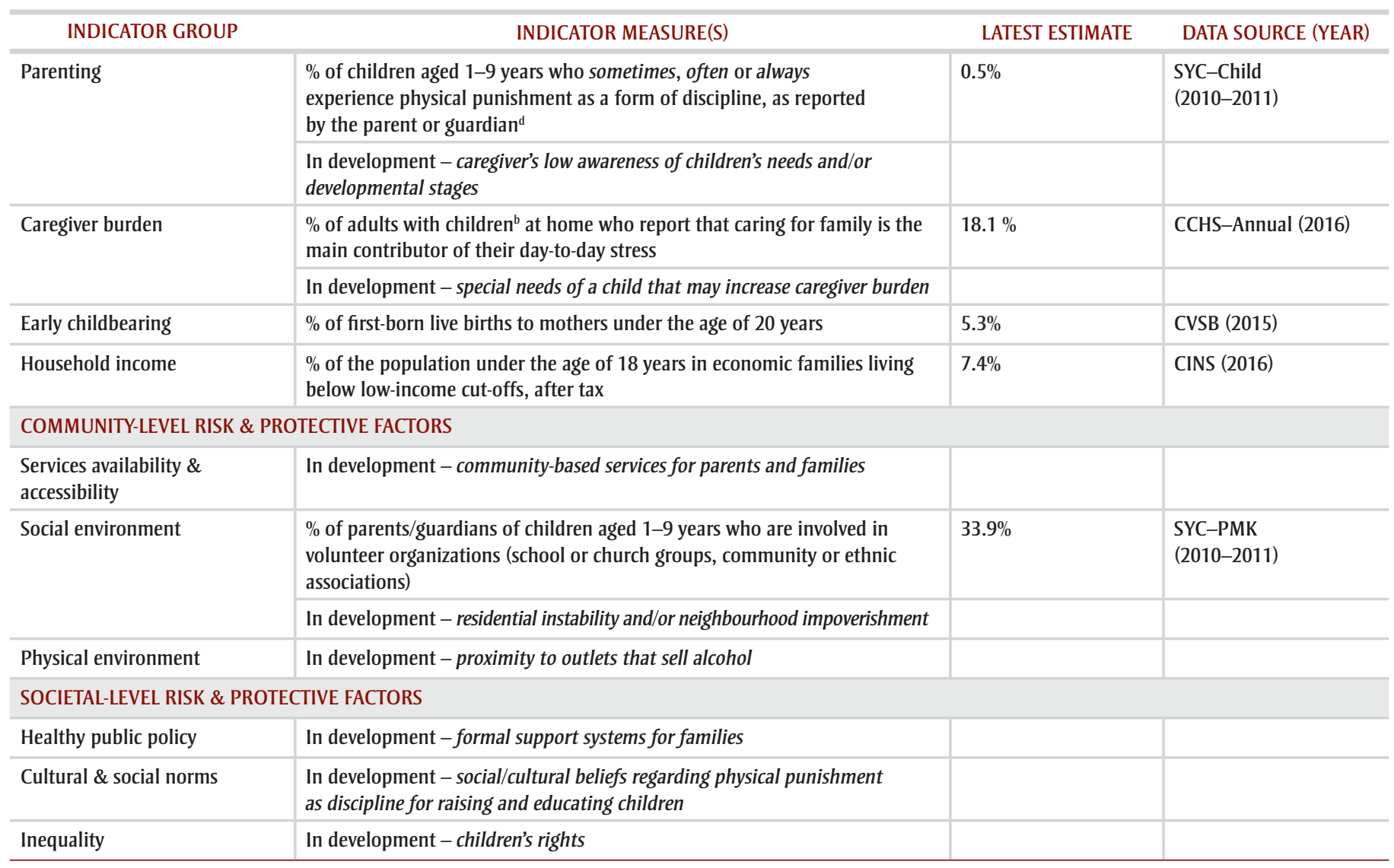

Abbreviations: CCHS-Annual, Canadian Community Health Survey - Annual Component; CCHS-Mental Health, Canadian Community Health Survey - Mental Health; CINS, Canadian Income Survey; CVSB, Canadian Vital Statistics - Birth Database (excluding Quebec); eCHIRPP, electronic Canadian Hospitals Injury Reporting and Prevention Program; ED, emergency department; GSS, General Social Survey; HBSC, Health Behaviour in School-aged Children; SYC-Child, Survey of Young Canadians - Child questionnaire; SYC-PMK, Survey of Young Canadians - Person Most Knowledgeable questionnaire; UCR, Uniform Crime Reporting Survey.

Note: "In development" refers to measures where their data source is currently not available or where more research is needed to identify a promising measure and data source.

${ }^{a}$ The estimates in this indicator framework were calculated in a manner consistent with those from the Statistics Canada report Family violence in Canada: a statistical profile, 2015, ${ }^{5}$ using a slightly different version of the database. All positive responses to all childhood maltreatment questions were counted as indicating maltreatment. In contrast, the original analysis rules for the Childhood Experiences of Violence Questionnaire (CEVQ) ${ }^{6}$ include all positive responses, except for two of the physical abuse subtypes (the less severe ones) and exposure to intimate partner violence if it occurred less than three times.

${ }^{\mathrm{b}} \mathrm{An}$ adult is defined as a person $18+$ years; a child is defined as a person under 18 years.

c Parental characteristics, not child characteristics, were considered as risk or protective factors for child maltreatment. This is based on a public health perspective that parental risk factors may represent potential outcomes of child maltreatment (such as prenatal substance abuse or violence).

"In the SYC-Child, the person answering these questions is confirmed at the beginning of the questionnaire to be the Person Most Knowledgeable (PMK) about the questions. "Parent/guardian" is used here for simplicity. 